\title{
QUANTUM LIMITS OF EISENSTEIN SERIES AND SCATTERING STATES
}

\author{
YIANNIS N. PETRIDIS, NICOLE RAULF, AND MORTEN S. RISAGER
}

\begin{abstract}
We identify the quantum limits of scattering states for the modular surface. This is obtained through the study of quantum measures of nonholomorphic Eisenstein series away from the critical line. We provide a range of stability for the quantum unique ergodicity theorem of Luo and Sarnak.
\end{abstract}

\section{INTRODUCTION}

An important problem of quantum chaos is to describe the limiting behaviour of eigenfunctions. On a compact negatively curved Riemannian manifold $X$ Shnirelman, Colin de Verdière, and Zelditch [18, 2, 22 have proved that for a 'generic' family of eigenfunctions $\left\{\phi_{j}\right\}$ of the Laplacian the associated measures $d \mu_{j}(z)=\left|\phi_{j}(z)\right|^{2} d \mu(z)$ converge weakly to the standard volume element $d \mu(z)$ of $X$, which we write as

$$
d \mu_{j}(z) \rightarrow d \mu(z) \text { as } j \rightarrow \infty .
$$

Zelditch [23] extended the result to finite volume hyperbolic surfaces. Lindenstrauss and Soundararajan [11, 19] have proved that for $X=\Gamma \backslash \mathbb{H}^{2}$ where $\Gamma \subset \mathrm{PSL}_{2}(\mathbb{Z})$ is of a certain arithmetic type (1.1) holds if $\phi_{j}$ runs through the set of Hecke-Maaß cusp forms. Earlier Luo and Sarnak 12 investigated the question of quantum chaos for Eisenstein series $E(z, 1 / 2+i t)$, i.e. generalized eigenfunctions on $X=$ $\mathrm{PSL}_{2}(\mathbb{Z}) \backslash \mathbb{H}^{2}$. Since this series is not square integrable a certain normalization is needed. The actual statement in 12 is the following: Let $A$ and $B$ be compact Jordan measurable subsets of $X$. Then

$$
\lim _{t \rightarrow \infty} \frac{\int_{A}|E(z, 1 / 2+i t)|^{2} d \mu(z)}{\int_{B}|E(z, 1 / 2+i t)|^{2} d \mu(z)}=\frac{\mu(A)}{\mu(B)} .
$$

In fact, see [12], this follows from the result

$$
\int_{A}|E(z, 1 / 2+i t)|^{2} d \mu(z) \sim \frac{6}{\pi} \cdot \mu(A) \log t, \quad t \rightarrow \infty .
$$

A general cofinite subgroup likely has few embedded eigenvalues, possibly finite, so that (1.1) may be irrelevant. So far the quantum unique ergodicity of Eisenstein series is unproven for a general cofinite subgroup. A good substitute for the embedded eigenvalues are the scattering poles (resonances). A natural question is to study the quantum limits of these scattering states. We address this question

Date: November 5, 2018.

2000 Mathematics Subject Classification. Primary 11F72; Secondary 58G25, 35P25.

Key words and phrases. Quantum Limits, Eisenstein series, Scattering poles.

The first author was supported by NSF grant DMS-0401318, the second author was supported by a grant from DAAD (German Academic Exchange Service), and the third author was supported by a grant from The Danish Natural Science Research Council. 
for $\Gamma=\mathrm{PSL}_{2}(\mathbb{Z})$. As these states are not in $L^{2}\left(\Gamma \backslash \mathbb{H}^{2}\right)$, some normalization is also needed. Consider a simple pole $\rho$ of the scattering matrix. By the explicit calculation of the scattering matrix, see (2.3), such a pole is equal to half a zero of the Riemann zeta function. The Eisenstein series has a pole at this point also and the residue has Fourier expansion

$$
\underset{s=\rho}{\operatorname{res}} E(z, s)=(\underset{s=\rho}{\operatorname{res}} \phi(s)) y^{1-\rho}+\sum_{m \neq 0} c_{m} \sqrt{y} K_{\rho-1 / 2}(2 \pi m y) e^{2 \pi i m x} .
$$

These scattering states are formal eigenfunctions of the Laplace operator. We choose to normalize them as follows: Set

$$
u_{\rho}(z)=(\underset{s=\rho}{\operatorname{res}} \phi(s))^{-1} \underset{s=\rho}{\operatorname{res}} E(z, s),
$$

so that the scattering functions have the simplest possible growth behaviour at infinity, namely $y^{1-\rho}$.

We let $\left\{\gamma_{n}\right\}$ be a sequence of zeroes of the Riemann zeta function with $1 / 2 \leq$ $\Re\left(\gamma_{n}\right)$ which satisfies $\lim _{n} \gamma_{n}=\gamma_{\infty}<1$. Automatically $\Re\left(\gamma_{n}\right)<1$ and the Riemann hypothesis is equivalent to $\gamma_{n}=\gamma_{\infty}=1 / 2$, but we shall not assume it. The points $\rho_{n}=\gamma_{n} / 2$ are poles of the scattering matrix $\phi(s)$.

Theorem 1.1. Let $A$ be a compact Jordan measurable subset of $X$. Then

$$
\int_{A}\left|u_{\rho_{n}}(z)\right|^{2} d \mu(z) \rightarrow \int_{A} E\left(z, 2-\gamma_{\infty}\right) d \mu(z)
$$

as $n \rightarrow \infty$. This means that the quantum limit of the measures $\left|u_{\rho_{n}}(z)\right|^{2} d \mu(z)$ is the invariant, absolutely continuous measure $E\left(z, 2-\gamma_{\infty}\right) d \mu(z)$.

Remark 1.2. We know that a positive proportion of the zeros $\rho$ of $\zeta(s)$ lie on the critical line and are simple. In fact, this proportion is at least $40.58 \%$, see 1]. Under the Riemann hypothesis and the conjectured simplicity of the Riemann zeros, there is only one quantum limit, the one described in the theorem above: $E(z, 3 / 2) d \mu(z)$.

Theorem 1.1 follows rather easily by studying the quantum limits of Eisenstein series off the critical line. We present two such theorems. The first addresses the stability of (1.2) if, instead of real spectral value $1 / 4+t^{2}$, we move in the complex plane. To be precise let

$$
d \mu_{s(t)}(z)=|E(z, s(t))|^{2} d \mu(z)
$$

where

$$
s(t)=\sigma_{t}+i t,
$$

$\sigma_{t}>1 / 2$. We investigate what happens in the limit as $t \rightarrow \infty$, assuming that $\sigma_{t} \rightarrow \sigma_{\infty} \geq 1 / 2$. We find qualitative differences depending on whether $\sigma_{\infty}=1 / 2$ or not. If $\sigma_{\infty}=1 / 2$ the situation is very similar to that of [12].

Theorem 1.3. Assume $\sigma_{\infty}=1 / 2$ and $\left(\sigma_{t}-1 / 2\right) \log t \rightarrow 0$. Let $A, B$ be compact Jordan measurable subsets of $X$. Then

$$
\frac{\mu_{s(t)}(A)}{\mu_{s(t)}(B)} \rightarrow \frac{\mu(A)}{\mu(B)}
$$

as $t \rightarrow \infty$. In fact we have

$$
\mu_{s(t)}(A) \sim \mu(A) \frac{6}{\pi} \log t .
$$


Theorem 1.3 implies that the quantum unique ergodicity of Eisenstein series holds in a quite big region in the complex plane (physical plane): for spectral value $\lambda$ tending to infinity, the results holds as long as $\Im(\lambda)=o(\sqrt{\Re(\lambda)} / \log \Re(\lambda))$ in the region $\Re(\lambda) \geq 0$. To see this we write $\lambda=s(1-s)$ with $s=\sigma+i t$ and $\sigma \geq 1 / 2$. Then $\Im(\lambda)=o(\sqrt{\Re(\lambda)} / \log \Re(\lambda))$ implies $\Re(\lambda)=\sigma(1-\sigma)+t^{2} \rightarrow \infty$. We easily deduce that $\sigma$ is bounded. Then $\left.(1-2 \sigma) t=o\left(\sqrt{\sigma(1-\sigma)+t^{2}}\right) / \log \left(\sigma(1-\sigma)+t^{2}\right)\right)$ gives $(\sigma-1 / 2) \log t \rightarrow 0$. So we can apply Theorem 1.3 .

Surprisingly the situation is qualitatively different when $\sigma_{\infty}>1 / 2$. We prove the following theorem:

Theorem 1.4. Assume $\sigma_{\infty}>1 / 2$. Let $A$ be a compact Jordan measurable subset of $X$. Then

$$
\mu_{s(t)}(A) \rightarrow \int_{A} E\left(z, 2 \sigma_{\infty}\right) d \mu(z)
$$

as $t \rightarrow \infty$.

This proves that, when $\sigma_{\infty}>1 / 2$, the measures $d \mu_{s(t)}$ do not become equidistributed. In fact it suggests in this case to consider different measures

$$
\nu_{s(t)}(z)=\left|\frac{E(z, s(t))}{\sqrt{E\left(z, 2 \sigma_{\infty}\right)}}\right|^{2} d \mu(z) .
$$

We note that, since $2 \sigma_{\infty}>1$, we have $E\left(z, 2 \sigma_{\infty}\right)>0$. The downside of this definition is that the function $E(z, s(t)) / \sqrt{E\left(z, 2 \sigma_{\infty}\right)}$ is not an eigenfunction of the Laplacian in contrast to $E(z, s(t))$. The upside is that the corresponding measures become equidistributed:

Corollary 1.5. Assume $\sigma_{\infty}>1 / 2$. Let $A$ be a compact Jordan measurable subset of $X$. Then

$$
\nu_{s(t)}(A) \rightarrow \mu(A), \quad t \rightarrow \infty .
$$

The result of Theorem 1.4 looks similar to Theorem 1 in [5], where the authors consider the equidistribution of Eisenstein series for convex co-compact subgroups $\Gamma$ of Iso $\left(\mathbb{H}^{n+1}\right)$ with Hausdorff dimension of the limit set $\delta_{\Gamma}$ satisfying $\delta_{\Gamma}<n / 2$. In both theorems the Eisenstein series $E\left(z, 2 \sigma_{\infty}\right)$ is well-defined and $E\left(z, 2 \sigma_{\infty}\right) d \mu(z)$ is the quantum limit.

Similar results to Theorem 1.1 and Theorem 1.4 for more general surfaces with cuspidal ends have recently been announced by Dyatlov [3].

Remark 1.6. The crucial ingredients in [12] are

(i) A subconvex estimate for the $L$-series of a Maaß cusp form on its critical line, e.g. $L\left(\phi_{j}, 1 / 2+i t\right) \ll(1+|t|)^{1 / 3+\epsilon}$, see [14].

(ii) A subconvex estimate for the Riemann zeta function on its critical line, e.g. $\zeta(1 / 2+i t) \ll(1+|t|)^{1 / 6+\epsilon}$.

(iii) Estimates for $\zeta(1+i t)$ and $\left(\zeta^{\prime} / \zeta\right)(1+i t)$.

For Theorem 1.3 above we use subconvex bounds on $L$-functions and $\zeta(s)$. When $\sigma_{\infty}>1 / 2$, i.e. in Theorem 1.4 only convexity bounds are used. While we use estimates on $\zeta(1+i t)$ and $1 / \zeta(1+i t)$ in both cases, the estimate for $\left(\zeta^{\prime} / \zeta\right)(1+i t)$ is required only for the theorem of Luo-Sarnak. Our results clarify the mechanism for quantum unique ergodicity of Eisenstein series. 
Remark 1.7. Equation (1.2) was extended by Jakobson [9] to the unit tangent bundle of $X$. Koyama [10] extended the result to Eisenstein series for $\mathrm{PSL}_{2}(\mathbb{Z}[i])$, and Truelsen [21] to Eisenstein series for $\operatorname{PSL}_{2}\left(\mathcal{O}_{K}\right)$, with $\mathcal{O}_{K}$ the integers of a totally real field $K$ of finite degree over $\mathbb{Q}$ with narrow class number one.

In both cases bounds of the type (ii), (iii), and (iii) above are used. In the case of $K=\mathbb{Q}(i)$ the subconvex estimate analogous to (ii) was established by Petridis and Sarnak 15, and the general case was established by Michel and Venkatesh 13. As a substitute of (iii) and (iii) one uses estimates for the Dedekind zeta function $\zeta_{K}$.

The analogous question for holomorphic Hecke cusp form of weight $k$ has recently been resolved by Holowinsky and Soundararajan [6]. Let $f_{k}$ be a sequence of $L^{2}$ normalized holomorphic Hecke cusp forms for the group $\mathrm{SL}_{2}(\mathbb{Z})$ of weight $k$ and let $\phi_{k}(z):=y^{k / 2} f_{k}(z)$. Then the measures $\left|\phi_{k}(z)\right|^{2} d \mu(z)$ converge weakly to $d \mu(z)$, as previously conjectured by Rudnick and Sarnak. We note that in this case $\phi_{k}$ is eigenfunction of the weight $k$ Laplacian with eigenvalue $k / 2(1-k / 2)$.

\section{Proofs}

The non-holomorphic Eisenstein series $E(z, s),(z, s) \in \mathbb{H}^{2} \times \mathbb{C}$ is defined for $\Re(s)>1$ by

$$
E(z, s)=\sum_{\gamma \in \Gamma_{\infty} \backslash \Gamma} \Im(\gamma z)^{s} .
$$

Here $\Gamma=\mathrm{PSL}_{2}(\mathbb{Z})$ and $\Gamma_{\infty}$ is the cyclic subgroup generated by $z \mapsto z+1$. The Eisenstein series $E(z, s)$ admits a Fourier expansion of the cusp $i \infty$, see e.g. 8,

$$
\begin{aligned}
E(z, s) & =\sum_{n \in \mathbb{Z}} a_{n}(y, s) e^{2 \pi i n x} \\
& =y^{s}+\phi(s) y^{1-s}+\frac{2 y^{1 / 2}}{\xi(2 s)} \sum_{n \neq 0}|n|^{s-1 / 2} \sigma_{1-2 s}(|n|) K_{s-1 / 2}(2 \pi|n| y) e^{2 \pi i n x} .
\end{aligned}
$$

Here $\xi(s)=\pi^{-s / 2} \Gamma(s / 2) \zeta(s)$ is the completed Riemann zeta function satisfying the functional equation $\xi(s)=\xi(1-s), \sigma_{c}(n)$ is the sum of the $c^{\prime}$ th powers of the divisors of $n$, and $K_{s}(y)$ is the $K$-Bessel function. The scattering matrix is

$$
\phi(s)=\frac{\xi(2-2 s)}{\xi(2 s)} .
$$

We notice that the corresponding expression in [12 is missing a factor of 2 in the non-zero terms. This is irrelevant for their purpose but becomes crucial for ours.

The spectral decomposition of $L^{2}\left(\Gamma \backslash \mathbb{H}^{2}\right)$ allows us to consider separately Maaß cusp forms and incomplete Eisenstein series.

2.1. Maaß cusp forms. Since there is a basis of the cuspidal eigenspaces consisting of Hecke-Maaß cusp forms, we restrict our attention to those.

Lemma 2.1. Let $\phi_{j}$ be a Hecke-Maaß cusp form. Then

$$
\int_{\Gamma \backslash \mathbb{H}^{2}} \phi_{j}\left|E\left(z, \sigma_{t}+i t\right)\right|^{2} d \mu(z) \rightarrow 0,
$$

as $t \rightarrow \infty$. 
Proof. The Maaß cusp form $\phi_{j}$ has a Fourier expansion

$$
\phi_{j}(z)=y^{1 / 2} \sum_{n \neq 0} \lambda(n) K_{i t_{j}}(2 \pi n y) e(n x),
$$

with $\lambda(1)=1$. We assume that it is even, since, if it is odd, $\left\langle\phi_{j}, \mu_{s(t)}\right\rangle=0$. Being a Hecke eigenform, $\phi_{j}$ has an $L$-series with Euler product

$$
L\left(\phi_{j}, s\right)=\sum_{n=1}^{\infty} \frac{\lambda(n)}{n^{s}}=\prod_{p}\left(1-\lambda(p) p^{-s}+p^{-2 s}\right)^{-1} .
$$

We want to understand the behavior as $t \rightarrow \infty$ of

$$
J_{j}(t)=\int_{\Gamma \backslash \mathbb{H}^{2}} \phi_{j}(z)|E(z, s(t))|^{2} d \mu(z) .
$$

We calculate

$$
I_{j}(s)=\int_{\Gamma \backslash \mathbb{H}^{2}} \phi_{j}(z) E(z, s(t)) E(z, s) d \mu(z),
$$

and set $s=\overline{s(t)}$ to recover $J_{j}(t)$. For fixed $s, I_{j}(s)$ is a holomorphic function of $w=s(t)$. In [12 this function is identified for $w=1 / 2+i t$, so we use the principle of analytic continuation to deduce that

$$
I_{j}(s)=\frac{R(s)}{\xi(2 s(t))} \frac{\prod \Gamma\left(\frac{s \pm i t_{j} \pm(s(t)-1 / 2)}{2}\right)}{2 \pi^{s} \Gamma(s)},
$$

with

$$
R(s)=\frac{L\left(\phi_{j}, s-s(t)+1 / 2\right) L\left(\phi_{j}, s+s(t)-1 / 2\right)}{\zeta(2 s)} .
$$

We plug $s=\overline{s(t)}$ to get

$$
J_{j}(t)=2^{-1} \pi^{s(t)-\overline{s(t)}} L\left(\phi_{j}, 1 / 2-2 i t\right) L\left(\phi_{j}, 2 \sigma_{t}-1 / 2\right) \frac{\prod \Gamma\left(\frac{\overline{s(t)} \pm i t_{j} \pm(s(t)-1 / 2)}{2}\right)}{|\Gamma(s(t)) \zeta(2 s(t))|^{2}} .
$$

We apply Stirling's formula [7, 5.112] in the form

$$
|\Gamma(\sigma+i t)|=\sqrt{2 \pi}|t|^{\sigma-1 / 2} e^{-\frac{\pi}{2}|t|}\left(1+O\left(|t|^{-1}\right)\right)
$$

uniformly for $|\sigma| \leq M$. Using this we find that the quotient of Gamma factors is $\ll_{j}|t|^{1 / 2-2 \sigma_{t}}$.

If $\sigma_{t}$ is bounded away from $1 / 2$, the function $|\zeta(2 s(t))|^{-2}$ is bounded and the convexity estimate $L\left(\phi_{j}, 1 / 2+i t\right) \ll t^{1 / 2}$ suffices to guarantee that $\lim J_{j}(t)=0$.

If $\sigma_{t}$ is not bounded away from $1 / 2$ and we need non-trivial estimates for $\zeta(2 s(t))^{-1}$ and $L\left(\phi_{j}, 1 / 2+i t\right)$ to reach the same conclusion. Such estimates are certainly available: the estimate

$$
\log ^{-1}|t| \ll|\zeta(2 s(t))| \ll \log |t|
$$

is classical in the theory of the Riemann zeta function (see [20, 3.6.5 and 3.11.8]), and the subconvexity estimate

$$
L\left(\phi_{j}, 1 / 2+i t\right)=O_{j, \epsilon}\left(|t|^{1 / 3+\epsilon}\right)
$$

was proved by Meurman [14. We note that any subconvexity estimate $L\left(\phi_{j}, 1 / 2+\right.$ $i t)=O\left(|t|^{1 / 2-\epsilon}\right)$ suffices to show that $\lim J_{j}(t)=0$. 
2.2. Incomplete Eisenstein series. We now concentrate on the contribution of the incomplete Eisenstein series. Let $h(y) \in C^{\infty}\left(\mathbb{R}^{+}\right)$be a function which decreases rapidly at 0 and $\infty$. This means that $h(y)=O_{N}\left(y^{N}\right)$ for $0<y \leq 1$ and $h(y)=$ $O\left(y^{-N}\right)$ for $y \gg 1$ for all $N \in \mathbb{N}$. Its Mellin transform is

$$
H(s)=\int_{0}^{\infty} h(y) y^{-s} \frac{d y}{y}
$$

and the Mellin inversion formula gives

$$
h(y)=\frac{1}{2 \pi i} \int_{a-i \infty}^{a+i \infty} H(s) y^{s} d s
$$

for any $a \in \mathbb{R}$. The function $H(s)$ is entire and $H(\sigma+i t)$ is in the Schwartz space in the $t$ variable for any $\sigma \in \mathbb{R}$. We consider the incomplete Eisenstein series

$$
F_{h}(z)=\sum_{\gamma \in \Gamma_{\infty} \backslash \Gamma} h(\Im(\gamma z))=\frac{1}{2 \pi i} \int_{a-i \infty}^{a+i \infty} H(s) E(z, s) d s .
$$

Lemma 2.2. Let $F_{h}$ be an incomplete Eisenstein series as above. Then

$$
\begin{aligned}
\int_{\Gamma \backslash \mathbb{H}^{2}} F_{h}(z)\left|E\left(z, \sigma_{t}+i t\right)\right|^{2} d & \mu(z) \sim \\
& \begin{cases}\int_{\Gamma \backslash \mathbb{H}^{2}} F_{h}(z) E\left(z, 2 \sigma_{\infty}\right) d \mu(z), & \text { if } \sigma_{\infty} \neq 1 / 2, \\
\int_{\Gamma \backslash \mathbb{H}^{2}} F_{h}(z) d \mu(z) 6 \pi^{-1} \log t, & \text { if }\left(\sigma_{t}-1 / 2\right) \log t \rightarrow 0,\end{cases}
\end{aligned}
$$

as $t \rightarrow \infty$.

Proof. We choose $a$ such that $a>2 \sigma_{t}$ for all $t$. The function $F_{h}(z)$ is smooth and rapidly decreasing in the cusp. Then unfolding and using Parseval we get

$$
\begin{aligned}
\int_{\Gamma \backslash \mathbb{H}^{2}} F_{h}(z) d \mu_{s(t)}(z) & =\int_{\Gamma \backslash \mathbb{H}^{2}} F_{h}(z)|E(z, s(t))|^{2} d \mu(z) \\
& =\int_{0}^{\infty} \int_{0}^{1} h(y)|E(z, s(t))|^{2} \frac{d x d y}{y^{2}} \\
& =\int_{0}^{\infty} h(y)\left(\sum_{n \in \mathbb{Z}}\left|a_{n}(y, s(t))\right|^{2}\right) \frac{d y}{y^{2}} .
\end{aligned}
$$

2.3. Contribution of the constant term. By (2.2) we have

$$
\left|a_{0}(y, s(t))\right|^{2}=y^{2 \sigma_{t}}+2 \Re\left(\phi(s(t)) y^{1-2 i t}\right)+|\phi(s(t))|^{2} y^{2-2 \sigma_{t}} .
$$

We analyze the three terms separately. The first term is

$$
\int_{0}^{\infty} h(y) y^{2 \sigma_{t}-1} \frac{d y}{y}=H\left(1-2 \sigma_{t}\right)
$$

which converges to $H\left(1-2 \sigma_{\infty}\right)$ when $t \rightarrow \infty$. Next

$$
\phi(s(t)) \int_{0}^{\infty} h(y) y^{-2 i t} \frac{d y}{y}=\phi(s(t)) H(2 i t) .
$$

The function $H(2 i t)$ decays rapidly and $\phi(s(t))$ is bounded, see [16, (8.6)]. By analyzing the same expression with $\overline{\phi(s(t))} y^{1-2 i t}$ instead of $\phi(s(t)) y^{1+2 i t}$ we find that the term in (2.13) involving $\Re\left(\phi(s(t)) y^{1-2 i t}\right)$ tends to zero. 
The last expression coming from the constant term is

$$
|\phi(s(t))|^{2} \int_{0}^{\infty} h(y) y^{1-2 \sigma_{t}} \frac{d y}{y}=|\phi(s(t))|^{2} H\left(2 \sigma_{t}-1\right) .
$$

Certainly $H\left(2 \sigma_{t}-1\right) \rightarrow H\left(2 \sigma_{\infty}-1\right)$, as $t \rightarrow \infty$ and $|\phi(s(t))|$ is bounded.

Using the explicit expression for $\phi(s)$ in (2.3) we have better control of the behavior of $\phi(s(t))$ when $\sigma_{\infty} \neq 1 / 2$. We have

$$
|\phi(s(t))|=\frac{|\xi(2-2 s(t))|}{|\xi(2 s(t))|}=\pi^{2 \sigma_{t}-1}\left|\frac{\zeta\left(2\left(1-\sigma_{t}\right)-2 i t\right)}{\zeta\left(2 \sigma_{t}+2 i t\right)}\right|\left|\frac{\Gamma\left(1-\sigma_{t}-i t\right)}{\Gamma\left(\sigma_{t}+i t\right)}\right| .
$$

Using the convexity bound $\zeta(\sigma+i t)=O\left(|t|^{(1-\sigma) / 2+\epsilon}\right)$ we get

$$
\zeta\left(2\left(1-\sigma_{t}\right)+i t\right)=O\left(|t|^{\sigma_{t}-1 / 2+\epsilon}\right) .
$$

By (2.8)

$$
\frac{1}{\zeta\left(2 \sigma_{t}+2 i t\right)}=O(\log |t|)
$$

The quotient of $\Gamma$-factors is asymptotic to $|t|^{1-2 \sigma_{t}}$ by (2.7). We therefore conclude that, when $\sigma_{\infty} \neq 1 / 2$, we have

$$
|\phi(s(t))| \rightarrow 0
$$

as $t \rightarrow \infty$.

To summarize we have proved that the contribution of the constant term in (2.13) converges to $H\left(1-2 \sigma_{\infty}\right)$ if $\sigma_{\infty} \neq 1 / 2$ and is $O(1)$ if $\sigma_{\infty}=1 / 2$.

2.4. Contribution of the non-constant terms. By (2.2) and (2.11) the contribution equals

$$
\begin{aligned}
A(t) & =\int_{0}^{\infty} \frac{1}{2 \pi i} \int_{\Re(s)=a} H(s) y^{s} d s \sum_{n=1}^{\infty} \frac{8 y}{|\xi(2 s(t))|^{2}} n^{2 \sigma_{t}-1}\left|\sigma_{1-2 s(t)}(n)\right|^{2}\left|K_{s(t)-1 / 2}(2 \pi n y)\right|^{2} \frac{d y}{y^{2}} \\
& =\int_{0}^{\infty} \frac{1}{2 \pi i} \int_{\Re(s)=a} H(s) \sum_{n=1}^{\infty} \frac{y^{s}}{(2 \pi n)^{s}} \frac{8}{|\xi(2 s(t))|^{2}} n^{2 \sigma_{t}-1}\left|\sigma_{1-2 s(t)}(n)\right|^{2}\left|K_{s(t)-1 / 2}(y)\right|^{2} d s \frac{d y}{y} \\
& =\frac{1}{2 \pi i} \int_{\Re(s)=a}^{H(s)} \frac{1}{(2 \pi)^{s}} \frac{8}{|\xi(2 s(t))|^{2}} \sum_{n=1}^{\infty} \frac{\left|\sigma_{1-2 s(t)}(n)\right|^{2}}{n^{s-\left(2 \sigma_{t}-1\right)}} \int_{0}^{\infty} y^{s}\left|K_{s(t)-1 / 2}(y)\right|^{2} \frac{d y}{y} d s .
\end{aligned}
$$

We now use [4, 6.576 (4)] to calculate the integral involving the $K$-Bessel functions, and the Ramanujan identity

$$
\sum_{n=1}^{\infty} \frac{\sigma_{a}(n) \sigma_{b}(n)}{n^{s}}=\frac{\zeta(s) \zeta(s-a) \zeta(s-b) \zeta(s-a-b)}{\zeta(2 s-a-b)}
$$

to see that

$$
\begin{aligned}
A(t) & =\frac{1}{2 \pi i} \int_{\Re(s)=a} H(s) \frac{1}{|\xi(2 s(t))|^{2}} \frac{\xi\left(s-2 \sigma_{t}+1\right) \xi(s-2 i t) \xi(s+2 i t) \xi\left(s+2 \sigma_{t}-1\right)}{\xi(2 s)} d s \\
& =\frac{1}{|\xi(2 s(t))|^{2} 2 \pi i} \int_{\Re(s)=a} B(s) d s,
\end{aligned}
$$


where $B(s)$ equal $H(s)$ times the $\xi$-factors. Since $\xi(s)$ has poles at $s=0,1$ the poles of $B(s)$ in the region $\Re(s) \geq 1 / 2$ are at $1 \pm 2 i t, 2 \sigma_{t}, 2-2 \sigma_{t}, \pm\left(2 \sigma_{t}-1\right)$ and $\pm 2 i t$. We now move the line of integration to $\Re(s)=1 / 2$. By considering the Stirling asymptotics for the $\Gamma$-factors, convexity bounds for the zeta functions, Eq. (2.8), and the rapid decay of $H(s)$ we see that $B(s)$ decays rapidly in vertical strips and this allows to move the line of integration. We find that

$$
\begin{aligned}
A(t)= & \frac{1}{|\xi(2 s(t))|^{2}}\left(\underset{s=1 \pm 2 i t}{\operatorname{res}} B(s)+\underset{s=2 \sigma_{t}}{\operatorname{res}} B(s)\right. \\
& \left.+\delta_{t} \cdot \underset{s=2-2 \sigma_{t}}{\operatorname{res}} B(s)+\left(1-\delta_{t}\right) \cdot \underset{s=2 \sigma_{t}-1}{\operatorname{res}} B(s)+\frac{1}{2 \pi i} \int_{\Re(s)=1 / 2} B(s) d s\right),
\end{aligned}
$$

with $\delta_{t}=1$ if $\sigma_{t}<3 / 4$ and 0 otherwise. We analyze these five terms.

(i) Using Stirling, convexity bounds on the zeta functions. (2.8) and the rapid decay of $H(1 \pm 2 i t)$ the term

$\frac{1}{|\xi(2 s(t))|^{2}} \operatorname{res}_{s=1 \pm 2 i t} B(s)=H(1 \pm 2 i t) \frac{\xi(1 \pm 4 i t) \xi\left(1 \pm 2 i t-2 \sigma_{t}+1\right) \xi\left(1 \pm 2 i t+2 \sigma_{t}-1\right)}{|\xi(2 s(t))|^{2} \xi(2 \pm 4 i t)}$

tends to zero as $t \rightarrow \infty$.

(ii) We now consider the second term:

$$
\frac{1}{|\xi(2 s(t))|^{2}} \underset{s=2 \sigma_{t}}{\operatorname{res}} B(s)=H\left(2 \sigma_{t}\right) \frac{\xi\left(4 \sigma_{t}-1\right)}{\xi\left(4 \sigma_{t}\right)}
$$

which converges to $H\left(2 \sigma_{\infty}\right) \frac{\xi\left(4 \sigma_{\infty}-1\right)}{\xi\left(4 \sigma_{\infty}\right)}$ when $t \rightarrow \infty$ and $\sigma_{\infty} \neq 1 / 2$. When $\sigma_{t} \rightarrow 1 / 2$ it behaves asymptotically like

$$
H(1) \frac{1}{4 \xi(2)\left(\sigma_{t}-1 / 2\right)}
$$

(iii) We then move on to the third term:

$$
\begin{aligned}
\frac{1}{|\xi(2 s(t))|^{2}} \operatorname{res}_{s=2-2 \sigma_{t}} B(s) & =H\left(2-2 \sigma_{t}\right) \frac{\xi\left(3-4 \sigma_{t}\right) \xi\left(2-2 \sigma_{t}-2 i t\right) \xi\left(2-2 \sigma_{t}+2 i t\right)}{|\xi(2 s(t))|^{2} \xi\left(4-4 \sigma_{t}\right)} \\
& =H\left(2-2 \sigma_{t}\right)|\phi(s(t))|^{2} \frac{\xi\left(3-4 \sigma_{t}\right)}{\xi\left(4-4 \sigma_{t}\right)}
\end{aligned}
$$

If $\sigma_{\infty} \neq 1 / 2$ we can use (2.14) to conclude that this tends to zero, and if $\sigma_{\infty}=1 / 2$ it behaves like

$$
H(1) \frac{-|\phi(s(t))|^{2}}{4 \xi(2)\left(\sigma_{t}-1 / 2\right)}+O(1)
$$

(iv) The fourth term is

$$
\frac{1}{|\xi(2 s(t))|^{2}} \operatorname{res}_{s=2 \sigma_{t}-1} B(s)=H\left(2 \sigma_{t}-1\right)|\phi(s(t))|^{2} .
$$

When $\sigma_{\infty} \neq 1 / 2$ this tends to zero and when $\sigma_{\infty}=1 / 2$ it is bounded, by the same arguments as for the third term. 
(v) We now deal with the last term, i.e.

$$
\begin{aligned}
\frac{1}{|\xi(2 s(t))|^{2} 2 \pi i} & \int_{\Re(s)=1 / 2} B(s) d s=\frac{1}{2 \pi\left|\xi\left(2 \sigma_{t}+2 i t\right)\right|^{2}} \\
& \times \int_{-\infty}^{\infty} H(1 / 2+i \tau) \frac{\left|\xi\left(1 / 2+2 \sigma_{t}-1+i \tau\right)\right|^{2} \xi(1 / 2+i(\tau-2 t)) \xi(1 / 2+i(\tau+2 t))}{\xi(1+2 i \tau)} d \tau .
\end{aligned}
$$

We note that $H(1 / 2+i \tau)$ is of rapid decay. We study first the exponential behaviour of the integral as a function of $t$. Stirling asymptotics for the integrand give:

$$
\left(e^{-\pi|\tau| / 4}\right)^{2} e^{-\pi|\tau / 2-t| / 2} e^{-\pi|\tau / 2+t| / 2}\left(e^{\pi|\tau| / 2}\right) \leq e^{-\pi t}
$$

which cancels with the exponential growth of $1 /|\xi(2 s(t))|^{2}$. Using (2.8), the rapid decay of $H(1 / 2+i \tau)$, and any polynomial bound in $\tau$ of $\zeta\left(2 \sigma_{t}-1 / 2+i \tau\right)$, we are reduced to estimate in $t$ the integral

$\frac{\log |t|}{\left(t^{-1 / 2+\sigma_{t}}\right)^{2}} \int_{-\infty}^{\infty} \tilde{H}(\tau)(1+|\tau+2 t|)^{-1 / 4}(1+|\tau-2 t|)^{-1 / 4}|\zeta(1 / 2+i(\tau-2 t)) \zeta(1 / 2+i(\tau+2 t))| d \tau$,

where $\tilde{H}$ is some function of rapid decay. We separate now the two cases $\sigma_{\infty}>1 / 2$ and $\sigma_{\infty}=1 / 2$. In the first case we use the convexity bound on the $\zeta$ function to estimate the expression as

$$
\frac{\log |t|}{\left(t^{-1 / 2+\sigma_{t}}\right)^{2}} \int_{-\infty}^{\infty} \tilde{H}(\tau)(1+|\tau+2 t|)^{\epsilon}(1+|\tau-2 t|)^{\epsilon} d \tau=o(1)
$$

as $\sigma_{\infty}>1 / 2$. For the second case we can use any subconvex bound $\zeta(1 / 2+i t)=$ $O\left(|t|^{1 / 4-\delta}\right)$, for instance Weyl's bound [20, Theorem 5.5]

$$
\zeta(1 / 2+i t) \ll|t|^{1 / 6+\epsilon} .
$$

We are reduced to estimate in $t$ the integral

$$
\begin{aligned}
& \frac{\log |t|}{\left(t^{-1 / 2+\sigma_{t}}\right)^{2}} \int_{-\infty}^{\infty} \tilde{H}(\tau)(1+|\tau+2 t|)^{-1 / 4+1 / 4-\delta}(1+|\tau-2 t|)^{-1 / 4+1 / 4-\delta} d \tau \\
& =t^{1-2 \sigma_{t}+\epsilon} \int_{-\infty}^{\infty} \tilde{H}(\tau)(1+|\tau+2 t|)^{-\delta}(1+|\tau-2 t|)^{-\delta} d \tau=o(1)
\end{aligned}
$$

This concludes the evaluation of the non-constant terms in (2.13).

To summarize we have proved that if $\sigma_{\infty} \neq 1 / 2$ the function $\int_{\Gamma \backslash \mathbb{H}^{2}} F_{h}(z) d \mu_{s(t)}(z)$ converges to

$$
H\left(1-2 \sigma_{\infty}\right)+H\left(2 \sigma_{\infty}\right) \frac{\xi\left(4 \sigma_{\infty}-1\right)}{\xi\left(4 \sigma_{\infty}\right)}
$$

as $t \rightarrow \infty$, and, if $\sigma_{\infty}=1 / 2$,

$$
\int_{\Gamma \backslash \mathbb{H}^{2}} F_{h}(z) d \mu_{s(t)}(z)=H(1) \frac{1-|\phi(s(t))|^{2}}{4 \xi(2)\left(\sigma_{t}-1 / 2\right)}+O(1),
$$


as $t \rightarrow \infty$. This finishes the proof once we notice that

$$
\begin{aligned}
H\left(1-2 \sigma_{\infty}\right)+ & H\left(2 \sigma_{\infty}\right) \frac{\xi\left(4 \sigma_{\infty}-1\right)}{\xi\left(4 \sigma_{\infty}\right)} \\
& =\int_{0}^{\infty} h(y)\left(y^{\left(2 \sigma_{\infty}-1\right)+1}+\phi\left(2 \sigma_{\infty}\right) y^{-2 \sigma_{\infty}+1}\right) \frac{d y}{y^{2}} \\
& =\int_{0}^{\infty} h(y)\left(\int_{0}^{1} E\left(z, 2 \sigma_{\infty}\right) d x\right) \frac{d y}{y^{2}} \\
& =\int_{\Gamma \backslash \mathbb{H}^{2}} F_{h}(z) E\left(z, 2 \sigma_{\infty}\right) d \mu(z)
\end{aligned}
$$

and

while

$$
H(1)=\int_{\Gamma \backslash \mathbb{H}^{2}} F_{h}(z) d \mu(z)
$$

$$
\frac{1-|\phi(s(t))|^{2}}{4 \xi(2)\left(\sigma_{t}-1 / 2\right)} \sim \frac{6}{\pi} \log t
$$

The last claim is seen as follows: By using the mean value theorem twice on the function $\sigma \mapsto \phi(\sigma+i t) \phi(\sigma-i t)$ we find

$$
\frac{1-|\phi(\sigma+i t)|^{2}}{1 / 2-\sigma}=\left(1-\left(1 / 2-\sigma^{\prime}\right)\left|\phi\left(\sigma^{\prime \prime}+i t\right)\right|^{2} \frac{\phi^{\prime}}{\phi}\left(\sigma^{\prime \prime} \pm i t\right)\right) \frac{\phi^{\prime}}{\phi}\left(\sigma^{\prime} \pm i t\right)
$$

for some $1 / 2 \leq \sigma^{\prime \prime} \leq \sigma^{\prime} \leq \sigma$, where $\frac{\phi^{\prime}}{\phi}(\sigma \pm i t)$ means $\frac{\phi^{\prime}}{\phi}(\sigma+i t)+\frac{\phi^{\prime}}{\phi}\left(\sigma^{\prime \prime}-i t\right)$. The claim now follows from $\left(\sigma_{t}-1 / 2\right) \log t \rightarrow 0$ and the well-known fact that

$$
\frac{\phi^{\prime}}{\phi}(\sigma \pm i t) \sim-4 \log t
$$

as $t \rightarrow \infty$ for $\sigma \geq 1 / 2$ and that $|\phi(\sigma+i t)|$ bounded for $\Re(s) \geq 1 / 2$ and $t>1$. The estimate (2.15) follows from the bounds on the zeta function [20, Theorem 5.17], combined with the Stirling asymptotics on the Gamma function.

It is straightforward to verify that Theorem 1.3. and Theorem 1.4 follow from Lemma 2.1 and Lemma 2.2 using an approximation argument like in the proof of [12, Proposition 2.3].

Proof of Theorem 1.1. We have

$$
\begin{aligned}
\left|u_{\rho_{n}}\right|^{2} d \mu(z) & =\left|\left(\underset{s=\rho_{n}}{\operatorname{res}} \phi(s)\right)^{-1} \underset{s=\rho_{n}}{\operatorname{res}} E(z, s)\right|^{2} d \mu(z) \\
& =\left|\left(\underset{s=\rho_{n}}{\operatorname{res}} \phi(s)\right)^{-1} \operatorname{res}_{s=\rho_{n}} \phi(s) E(z, 1-s)\right|^{2} d \mu(z) \\
& =\left|E\left(z, 1-\rho_{n}\right)\right|^{2} d \mu(z) .
\end{aligned}
$$

The result now follows from Theorem 1.4 with $\sigma_{\infty}=1-\gamma_{\infty} / 2$.

Proof of Corollary 1.5. Let $f$ be a test function for the convergence in Corollary 1.5. Then we use

$$
\frac{f(z)}{E\left(z, 2 \sigma_{\infty}\right)}
$$


as test function for Theorem 1.4 to deduce that, as $t \rightarrow \infty$

$$
\int_{\Gamma \backslash \mathbb{H}^{2}} \frac{f(z)}{E\left(z, 2 \sigma_{\infty}\right)} d \mu_{s(t)} \rightarrow \int_{\Gamma \backslash \mathbb{H}^{2}} \frac{f(z)}{E\left(z, 2 \sigma_{\infty}\right)} E\left(z, 2 \sigma_{\infty}\right) d \mu(z)=\int_{\Gamma \backslash \mathbb{H}^{2}} f(z) d \mu(z) .
$$

Finally one uses the approximation argument in [12, Proposition 2.3] to complete the proof.

\section{REFERENCES}

[1] H. Bui, J.B. Conrey and M. Young, More than 41\% of the zeros of the zeta function are on the critical line, arXiv:1002.4127v2 [math.NT].

[2] Y. Colin de Verdière, Ergodicité et fonctions propres du laplacien. Comm. Math. Phys. 102 (1985), no. 3, 497-502.

[3] S. Dyatlov, Quantum ergodicity of Eisenstein functions at complex energies, arXiv:1109.3338v1.

[4] I. S. Gradshteyn, I. M. Ryzhik, Table of Integrals, Series and Products, Fifth edition, Alan Jeffrey, ed., Academic Press, San Diego, 1994.

[5] C. Guillarmou, F. Naud, Equidistribution of Eisenstein series on convex co-compact hyperbolic manifolds, arXiv:1107.2655v1.

[6] R. Holowinsky, K. Soundararajan, Mass equidistribution for Hecke eigenforms. Ann. of Math. (2) 172 (2010), no. 2, 1517-1528.

[7] H. Iwaniec E. Kowalski, Analytic number theory. American Mathematical Society Colloquium Publications, 53. American Mathematical Society, Providence, RI, 2004. xii+615 pp.

[8] H. Iwaniec. Spectral methods of automorphic forms. Second edition. Graduate Studies in Mathematics, 53. American Mathematical Society, Providence, RI; Revista Matemtica Iberoamericana, Madrid, 2002. xii+220 pp.

[9] Jakobson, Dmitry Quantum unique ergodicity for Eisenstein series on $\mathrm{PSL}_{2}(\mathbb{Z}) \backslash \mathrm{PSL}_{2}(\mathbb{R})$. Ann. Inst. Fourier (Grenoble) 44 (1994), no. 5, 1477-1504.

[10] S. Koyama, Quantum ergodicity of Eisenstein series for arithmetic 3-manifolds. Comm. Math. Phys. 215 (2000), no. 2, 477-486.

[11] E. Lindenstrauss, Invariant measures and arithmetic quantum unique ergodicity. Ann. of Math. (2) 163 (2006), no. 1, 165-219.

[12] W. Luo, P. Sarnak, Quantum Ergodicity of Eigenfunctions on $P S L_{2}(\mathbb{Z}) \backslash \mathbb{H}^{2}$, Inst. Hautes Études Sci. Publ. Math. No. 81 (1995), 207-237.

[13] P. Michel , A. Venkatesh, The subconvexity problem for $\mathrm{GL}_{2}$. Publ. Math. Inst. Hautes tudes Sci. No. 111 (2010), 171-271.

[14] T. Meurman, On the order of the Maass L-function on the critical line. Number theory, Vol. I, Budapest, 1987, Colloq. Math. Soc. Janos Bolyai, 51 (1990), 325-354.

[15] Y. Petridis, P. Sarnak, Quantum unique ergodicity for $S L_{2}(\mathcal{O}) \backslash \mathbb{H}^{3}$ and estimates for Lfunctions. Dedicated to Ralph S. Phillips. J. Evol. Equ. 1 (2001), no. 3, 277-290.

[16] A. Selberg, Harmonic Analysis, Göttingen lecture notes, Collected papers Vol I, Springer Verlag.

[17] G. Shimura, The special values of the zeta functions associated with cusp forms. Comm. Pure Appl. Math. 29 (1976), no. 6, 783-804.

[18] A. Shnirelman, Ergodic properties of eigenfunctions. Uspehi Mat. Nauk 29 (1974), no. $6(180), 181-182$.

[19] K. Soundararajan, Quantum unique ergodicity for $S L_{2}(\mathbb{Z}) \backslash \mathbb{H}$. Ann. of Math. (2) 172 (2010), no. 2, 1529-1538.

[20] E. Titchmarsh, The theory of the Riemann zeta-function, Second Edition, Oxford University Press, London, 1986.

[21] J. L Truelsen, Quantum unique ergodicity of Eisenstein series on the Hilbert modular group over a totally real field. Forum Mathematicum, Vol. 23, No. 5, 891-931.

[22] S. Zelditch, Uniform distribution of eigenfunctions on compact hyperbolic surfaces. Duke Math. J. 55 (1987), no. 4, 919-941. 
[23] S. Zelditch, Selberg trace formulae and equidistribution theorems for closed geodesics and Laplace eigenfunctions: finite area surfaces. Mem. Amer. Math. Soc. 96 (1992), no. 465, vi+102 pp.

Department of Mathematics, University College London, Gower Street, London WC1E 6BT, United Kingdom

E-mail address: i.petridis@ucl.ac.uk

Laboratoire Paul Painlevé, U.F.R. de Mathématiques, Université Lille 1 Sciences et Technologies, 59655 Villeneuve D'Ascq Cédex, France

E-mail address: raulf@math.univ-lille1.fr

Department of Mathematical Sciences, University of Copenhagen, Universitetsparken 5, 2100 Copenhagen $\varnothing$, Denmark

E-mail address: risager@math.ku.dk 